\title{
Mode-resolved directional enhancement of spontaneous emission inside/outside finite multilayer hyperbolic metamaterials
}

\author{
Mahmoodi, Maryam; Tavassoli, Seyed Hassan; Lavrinenko, Andrei V.
}

Published in:

Materials Today Communications

Link to article, DOI:

10.1016/j.mtcomm.2019.100859

Publication date:

2020

Document Version

Early version, also known as pre-print

Link back to DTU Orbit

Citation (APA):

Mahmoodi, M., Tavassoli, S. H., \& Lavrinenko, A. V. (2020). Mode-resolved directional enhancement of spontaneous emission inside/outside finite multilayer hyperbolic metamaterials. Materials Today

Communications, 23, [100859]. https://doi.org/10.1016/j.mtcomm.2019.100859

\section{General rights}

Copyright and moral rights for the publications made accessible in the public portal are retained by the authors and/or other copyright owners and it is a condition of accessing publications that users recognise and abide by the legal requirements associated with these rights.

- Users may download and print one copy of any publication from the public portal for the purpose of private study or research.

- You may not further distribute the material or use it for any profit-making activity or commercial gain

- You may freely distribute the URL identifying the publication in the public portal 


\title{
Mode-resolved Directional Enhancement of Spontaneous Emission Inside/Outside Finite Multilayer Hyperbolic Metamaterials
}

\author{
Maryam Mahmoodia , Seyed Hassan Tavassoli, ${ }^{a, *}$, Andrei V. Lavrinenko ${ }^{b}$ \\ a Laser and Plasma Research Institute, Shahid Beheshti University, Velenjak, Tehran, Iran \\ ${ }^{b}$ Technical University of Denmark, Dept. of Photonics Engineering, Ørsteds plads, bldg. 345V, 2800 Kgs. Lyngby, Denmark
}

\begin{abstract}
The concept of large photonic density of states associated with high-k modes of Hyperbolic Metamaterials (HMMs) plays a significant role in the interests behind the HMM notion. Despite the theoretical predictions, it is proved that photonic Local Density of States (LDOS) is not infinite in practical multilayer HMMs. In the present study, a developed theory based on the effective medium approximation is used to probe the finite number of high-k modes in finite multilayer HMMs. The High-k modes are classified in two categories as short-range and long-range propagating modes, strongly dependent on the number of periods. Introducing a mode-resolved transition rate enhancement factor, emission coupling of a quantum emitter, placed inside and in near vicinity of HMM slabs, to the high-k modes is calculated. The results show that the short-range and long-range modes have the commanding influence on transition rate enhancement outside and inside the slabs, respectively. The results point out that HMM slabs having lower number of periods provide higher total LDOS giving rise to larger transition rate enhancement. The results prove the capability of engineering photonic density of states in finite HMMs and pave the way for application-orientated HMMs design.
\end{abstract}

Keywords: Local Photonic Density of States, Spontaneous Emission Transition Rate, Hyperbolic Metamaterials, High-k Modes

\section{Introduction}

Hyperbolic Metamaterials (HMMs) hold promise to embody the boundless possibilities in light-matter interaction on nanoscale[1]. Artificial materials having hyperbolic dispersion have been designed for various applications such as nano focusing [2], sensing [3, 4], wave front manipulation [5], sub diffraction imaging [6, 7], emission enhancement of quantum emitters [8, 9] and many more. HMMs have attracted a great attention for spontaneous emission enhancement due to the large photonic Local Density of States (LDOS) associated with their unique modes having large wave vector, so called high$k$ modes 10 11. The significance of LDOS in quantum photonics is evidenced in the Fermi's golden rule expressing that manipulation of photonic local density of states can change the spontaneous emission lifetime (transition rate) of a Quantum Emitter (QE) [12]. Directional propagation of the high-k modes is proved as another unique feature of the hyperbolic metamaterials [13] promising for improving emission collection of the QEs coupled to the HMM modes[14]. Artificial materials having nanometer-size building blocks like multilayer stacks [15, 16, 17] and nanowires [18, 19] in hyperbolic regime, have shown capabilities in engineering directional enhancement of quantum emitters spontaneous emission [20].

Based on the conventional Effective Medium Approximation (EMA)[21], the open form iso-frequency surface (ideal hyperboloid) of the HMMs have no upper limit for the wave vector of propagating waves. It leads to the conclusion that HMMs could

\footnotetext{
*h-tavassoli@sbu.ac.ir
}

provide infinite density of states. Yet, in practice, the constrains imposed by thickness of the building blocks and nonlocal effects proved that the LDOS of HMMs must be finite [22, 23]. Affiliation of LDOS to the high-k modes and the relevance of such modes to the number of periods in multilayer structures[24], creates an urge to study the individual high-k modes in finite thickness structures. Since the number of periods in HMM structures is a very practical question, an analysis of contribution of individual modes of finite thickness HMMs in total LDOS is needed to investigate the necessary number of periods required for providing a large LDOS in application-orientated multilayer HMMs. While the Green's function calculations 25, 26] and reflection coefficients of multilayer structures [27 28] as largely performed methods in literature do not provide an insight into the individual high-k modes LDOS, here we report the first demonstration of a mode-resolved LDOS and propagation direction calculation in multilayer hyperbolic metamaterials. To account for the practical finite number of periods, a theory based on the optical waveguide rules is developed for the finite thickness HMM slabs homogenized using a nonlocal EMA. We managed to find the dispersion relations of the individual high$k$ modes in any practical multilayer structures. Two classes of high-k modes in HMMs are distinguished and named as shortrange (SR) and long-range (LR) propagating modes. A quantum emitter is considered inside and nearby the HMM slabs to investigate contribution of the LR and SR high-k modes to transition rate enhancement and directionality of the spontaneous emission using a pragmatic theoretical approach. Our findings demonstrate that the lower the number of period is, the larger the LDOS of HMM slabs will be. Meanwhile, the SR 
modes provide larger enhancement factors for QEs placed outside the slabs and the LR modes take the role for QEs located inside the HMM slabs.

\section{Theory}

\subsection{High-k modes in finite HMMs}

Multilayer hyperbolic metamaterials are depicted in subwavelength periodic meta-dielectric layers with complex permittivity $\varepsilon_{\mathrm{m}}, \varepsilon_{\mathrm{d}}$ and thickness $t_{\mathrm{m}}, t_{\mathrm{d}}(<<\lambda)$, respectively. The conventional effective medium approximation is widely used to predict the HMMs optical behavior by a uniaxial permittivity tensor[29] with ordinary and extraordinary components as

$$
\varepsilon=\left[\begin{array}{ccc}
\varepsilon_{o} & 0 & 0 \\
0 & \varepsilon_{o} & 0 \\
0 & 0 & \varepsilon_{e}
\end{array}\right]
$$

It is proved that nonlocal effects in nanometer-size structures is considerable and drastically affects the precision of theoretical studies[22, 30, 31]. Thus, in utilizing effective medium approximation, a nonlocal homogenization should be taken into consideration. The operator effective medium theory 32 considers a power series dependency of the primitivity components on $k_{0} t$ to account for the nonlocality, where $k_{0}$ is the vacuum wavenumber and $p$ is the metal fill fraction in the unit cell of thickness $t=t_{\mathrm{m}}+t_{\mathrm{d}}$. For a bilayer unit cell of metaldielectric, the operator approach EMT is used in the present paper with the following nonlocal permittivity components taken up to $\left(k_{0} t\right)^{2}$.

$$
\begin{gathered}
\varepsilon_{\mathrm{o}}^{\mathrm{nl}}=\varepsilon_{\mathrm{o}}+\frac{\left(k_{0} t\right)^{2}}{6} \sigma \varepsilon_{\|} f(\beta) \\
\varepsilon_{\mathrm{e}}^{\mathrm{nl}}=\left\{\frac{1}{\varepsilon_{\mathrm{e}}}+\frac{\left(k_{0} t\right)^{2}}{6} \sigma\left(\frac{2 p-1}{\varepsilon_{\mathrm{r}}}-\frac{f(\beta)}{\varepsilon_{\perp}}\right)\right\}^{-1}
\end{gathered}
$$

The familiar extraordinary $\varepsilon_{\mathrm{e}}$ and ordinary $\varepsilon_{\mathrm{o}}$ local permittivity components as well as the other parameters are defined below, where contributing the metal's complex permittivity generates complex values.

$$
\begin{aligned}
\varepsilon_{\mathrm{o}} & =p \varepsilon_{\mathrm{m}}+(1-p) \varepsilon_{\mathrm{d}} & \varepsilon_{\mathrm{e}} & =\left(\frac{p}{\varepsilon_{\mathrm{m}}}+\frac{1-p}{\varepsilon_{\mathrm{d}}}\right)^{-1} \\
\varepsilon_{\|} & =p \varepsilon_{\mathrm{m}}-(1-p) \varepsilon_{\mathrm{d}} & \varepsilon_{\perp} & =\left(\frac{p}{\varepsilon_{\mathrm{m}}}-\frac{1-p}{\varepsilon_{\mathrm{d}}}\right)^{-1} \\
\varepsilon_{\mathrm{r}} & =\left(\frac{1}{\varepsilon_{\mathrm{m}}}+\frac{1}{\varepsilon_{\mathrm{d}}}\right)^{-1} & \sigma & =p(1-p)\left(\varepsilon_{\mathrm{d}}-\varepsilon_{\mathrm{m}}\right) \\
f(\beta) & =\frac{\beta^{2}}{\varepsilon_{\mathrm{r}}}-1 & &
\end{aligned}
$$

In a cartesian system that z-axis coincides with the optical axis of multilayer HMMs, the well-known dispersion equation of HMMs 21] considering the nonlocal parameters is written as

$$
\frac{k_{\mathrm{x}}^{2}+k_{\mathrm{y}}^{2}}{\varepsilon_{\mathrm{e}^{n l}}}+\frac{k_{\mathrm{z}}^{2}}{\varepsilon_{\mathrm{o}^{n l}}}=k_{0}^{2}
$$

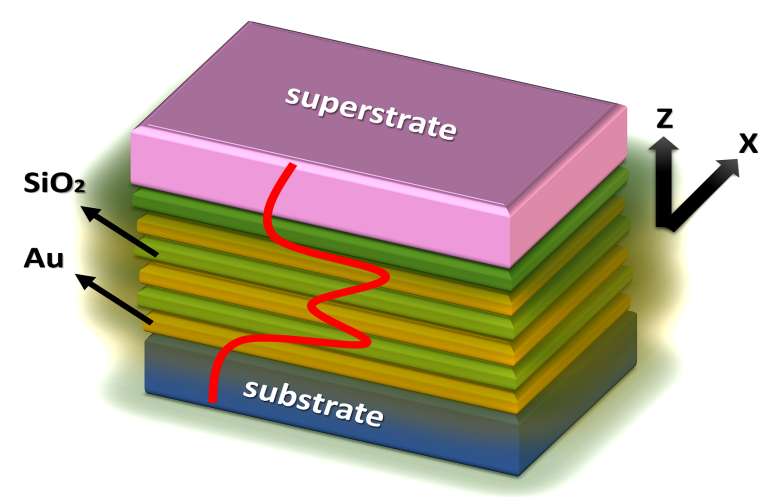

Figure 1: Finite thickness multilayer HMMs considered as waveguide slabs confining the propagating high-k modes.

Due to symmetry in $x$ and $y$ axes, without loss of generality, the wave vector $\mathbf{k}$ is taken as $\mathbf{k}=\beta k_{0} \hat{\mathbf{x}}+k_{\mathrm{z}} \hat{\mathbf{z}}$, where $\beta=$ $\frac{k_{\mathrm{x}}}{k_{0}}$ is the normalized transverse wavevector. Thus, the vertical component of the wavevector, $k_{\mathrm{z}}$, can be considered as

$$
k_{\mathrm{z}}=k_{0} \sqrt{-\varepsilon_{\mathrm{o}}^{\mathrm{nl}}\left(\frac{\beta^{2}}{\varepsilon_{\mathrm{e}}^{\mathrm{nl}}}-1\right)}
$$

Generally speaking, $k_{\mathrm{z}}$ is a complex parameter. As the nonlocal permittivities are dependent on $\beta$, the $k_{\mathrm{z}}$ of high-k waves in the HMMs will be a nonlinear function of $\beta$. Based on the relation between $\varepsilon_{\mathrm{e}}^{\mathrm{nl}}$ and $\beta$, for HMMs type II with the condition of $\varepsilon_{\mathrm{o}}^{\mathrm{nl}}<0$ and $\varepsilon_{\mathrm{e}}^{\mathrm{nl}}>0$, two classes of propagating waves can be conceived as

- Long-Range (LR) high-k waves

The $k_{\mathrm{z}}$ of these waves will be largely a real value under the condition of $\varepsilon_{\mathrm{e}}^{\mathrm{nl}}<\beta^{2}$. Therefore, the high-k waves will be propagating along the $z$ axis with a minor damping caused by the imaginary part.

- Short-Range (SR) high-k waves

Under the condition of $\varepsilon_{\mathrm{e}}^{\mathrm{nl}}>\beta^{2}$ large imaginary part of $k_{\mathrm{z}}$ results in a high damping for the bulk waves and leads to the kind of waves having short propagation length in the HMM.

To take into account the limited number of periods or equivalently limited thickness of the effective medium, we need to consider the boundary effects. Thus, the effective medium calculated out of the multilayer structure is deliberated as a finite thickness single layer sandwiched between two semi-infinite media as substrate and superstrate shown in Fig. 1. Here, the middle homogenous and uniaxial layer is considered as a waveguide for the SR and LR propagating high-k waves, being confined within the slab and attenuating outside in the superstrate and substrate. Defining $\beta$ as the effective index of the propagating waves inside the HMM slabs, the constrain of $\sqrt{\varepsilon_{1}} \leqslant \sqrt{\varepsilon_{2}}<\beta$ is satisfied for the asymmetric optical slab waveguide; $\varepsilon_{1} \leqslant \varepsilon_{2}$ is taken for simplicity. Solving Maxwellequations for the three homogenous layers, dispersion equations of high-k modes in finite thickness HMMs is calculated 


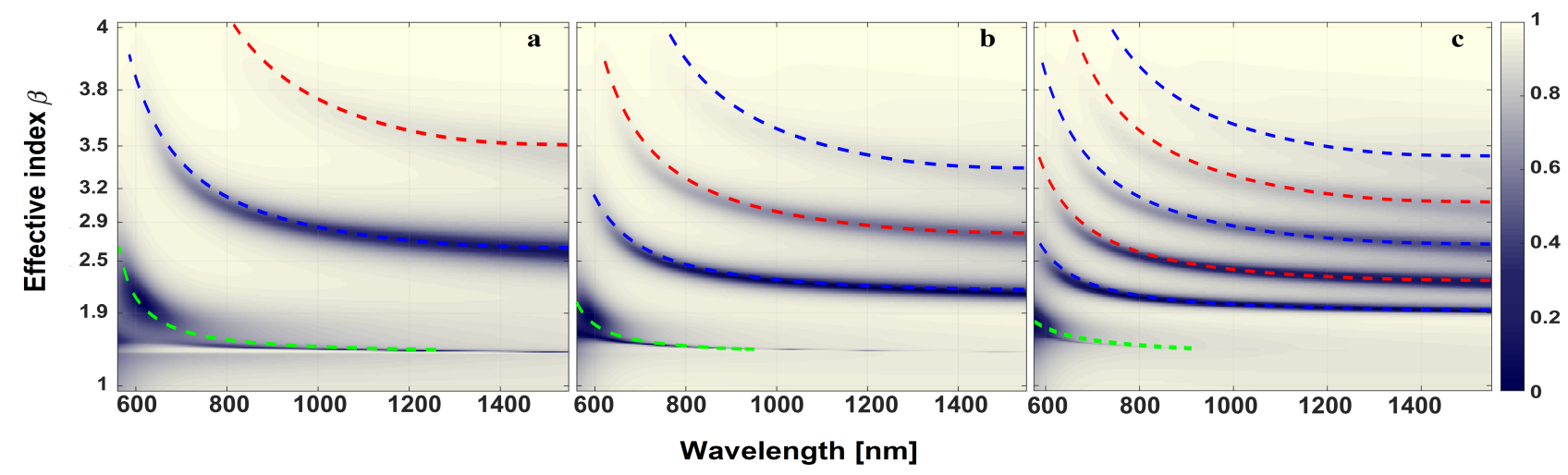

Figure 2: Bulk high-k modes dispersion in $\mathrm{HMM}$ slabs consisting of a) 3, b) 5, c) 8 periods of $15-25$ nm thick $\mathrm{Au}_{-} \mathrm{SiO}_{2}$ layers. TMM calculations in the background, theoretical SR high-k modes in green, even and odd LR modes in blue and red dashed lines, respectively.

as;

$$
\begin{gathered}
\tanh \left(\mathbb{K}_{\mathrm{SR}} k_{0} h\right)=\frac{\mathbb{K}_{\mathrm{SR}}\left(\varepsilon_{1} \mathbb{K}_{2}+\varepsilon_{2} \mathbb{K}_{1}\right)}{\frac{\varepsilon_{1} \varepsilon_{2}}{-\varepsilon_{\mathrm{o}}^{\mathrm{n}}} \mathbb{K}_{\mathrm{SR}}^{2}-\varepsilon_{\mathrm{o}}^{\mathrm{nl}} \mathbb{K}_{1} \mathbb{K}_{2}} \\
\tan \left(\mathbb{K}_{\mathrm{LR}} k_{0} h\right)=\frac{\mathbb{K}_{\mathrm{LR}}\left(\varepsilon_{1} \mathbb{K}_{2}+\varepsilon_{2} \mathbb{K}_{1}\right)}{\frac{\varepsilon_{1} \varepsilon_{2}}{\varepsilon_{\mathrm{o}}^{\mathrm{n}}} \mathbb{K}_{\mathrm{LR}}^{2}-\varepsilon_{\mathrm{o}}^{\mathrm{nl}} \mathbb{K}_{1} \mathbb{K}_{2}}
\end{gathered}
$$

where $h$ stands for the thickness of slab, $h=n \times t$, here $n$ is the number of period. $\mathbb{K}_{1}=\sqrt{\beta^{2}-\varepsilon_{1}}$ and $\mathbb{K}_{2}=\sqrt{\beta^{2}-\varepsilon_{2}}$ are the normal components (in the $z$ direction) of the normalized wavevectors of evanescent waves in the substrate and superstrate, $\mathbb{K}_{\mathrm{SR}}=\sqrt{-\varepsilon_{\mathrm{o}}^{\mathrm{nl}}\left(1-\frac{\beta^{2}}{\varepsilon_{\mathrm{e}^{\mathrm{n} l}}}\right)}$ and $\mathbb{K}_{\mathrm{LR}}=\sqrt{-\varepsilon_{\mathrm{o}}^{\mathrm{nl}}\left(\frac{\beta^{2}}{\varepsilon_{\mathrm{e}^{\mathrm{nl}}}}-1\right)}$ are those of SR and LR high-k modes in the HMM slab respectively[24].

\subsection{Mode-resolved Photonic Local Density of States}

Many studies in literature are reported on multilayer HMMs application in spontaneous emission enhancement of quantum emitters placed inside [14] and outside [16, 33] the structures. Fabricating emitters in precise locations from practical point of view is a challenging procedure that make the need for a comprehensive study on the location of emitters for better performance. Besides that, out coupling of emission coupled to the HMM modes requires some extraction methods such as grating [34] and nanopatterning of the HMMs [9]. In all cases identifying the wave vector of the mode which is coupled to emitter is necessary for designing the extraction method. Here, realizing the number and the nature of high-k modes in finite thickness HMMs, we aim to compute a mode-resolved local density of states using an analytical formula to calculate the LDOS portion of each HMMs waveguide mode; contributing in the transition rate of an emitter placed nearby and inside the HMM slabs. Generally speaking, radiation occurs from an atom whose nucleus is localized in space. Typically, the wavelength of light emitted by an atom is several orders of magnitude greater than the spatial extend of the electron orbits about the nucleus. Therefore, the emission occurring can be thought of redistribution of charges (dipole formation) in the emitter during the transition. In the spontaneous emission process, the electric dipole moment plays the strongest and main contributing part. In this regard, an electric dipole is chosen as an emitter for the furthur theoretical investigations. From the Fermi's golden rule developed for a slab waveguide[35], the transition rate of an emitter is given by

$$
\gamma_{w g}(\omega)=2 \pi\left|g\left(r_{e d}, \omega\right)\right|^{2} D_{2 D}(\omega)
$$

Here $D_{2 D}(\omega)$ is defined as the local density of states for each mode of the slab in 2D space and $\left|g\left(r_{e d}, \omega\right)\right|^{2}$ is the coupling strength between the mode and the dipole at position $r_{e d}$, in a medium of refractive index $\sqrt{\epsilon}_{e d}[36]$,

$$
\begin{array}{r}
\left|g\left(r_{e d}, \omega\right)\right|^{2}=\frac{\omega\left|\mu_{0}\right|^{2}}{2 \hbar \epsilon_{e d} \epsilon_{0} V_{e f f}} \\
D_{2 D}=\frac{l^{2} \omega}{2 \pi V_{g}(\omega) V_{p}(\omega)},
\end{array}
$$

where $V_{p h}$ and $V_{g r}$ stand for the phase and group velocity of high-k waveguide modes and are calculated based on $V_{p h}=\frac{c}{\beta}$ and $V_{g r}=V_{p h}-\lambda \frac{\partial V_{p v}}{\partial \lambda}$. Here, $\beta$ is obtained from solving the HMM's dispersion relations derived in Eq. (5). $V_{\text {eff }}=l^{2} L_{e f f}$ is the effective mode volume assigned to each high-k mode where $l$ is an arbitrary quantization length in the $x-y$ plane and $L_{e f f}$ is the effective mode length in the $z$ direction defined as

$$
L_{e f f}=\frac{1}{2} \frac{\int_{-\infty}^{\infty}\left[\epsilon(z)|E(z, \omega)|^{2}\right] d z}{\epsilon_{e d}\left|E\left(z_{e d}, \omega\right)\right|^{2}}
$$

To find the transition rate Enhancement Factor, $E F$, the transition rate of a QE coupled to each HMM mode, $\gamma_{w g}(\omega)$, needs to be compared to that of the isotropic media, $\gamma_{i s o}(\omega)=$ $\frac{\sqrt{\epsilon_{e d}} \omega^{3}\left|\mu_{0}\right|^{2}}{3 \hbar \pi \epsilon_{0} c^{3}}$. In case the QE is located outside the slab, the enhancement factor do not necessarily indicate that QE emission into HMM modes prevail the modes in the other half space. Therefore, another coefficient as coupling efficiency or Coupling Factor, $C F$ needs to be defined as the followings.

$$
E F=\frac{\gamma_{w g}(\omega)}{\gamma_{i s o}(\omega)}
$$



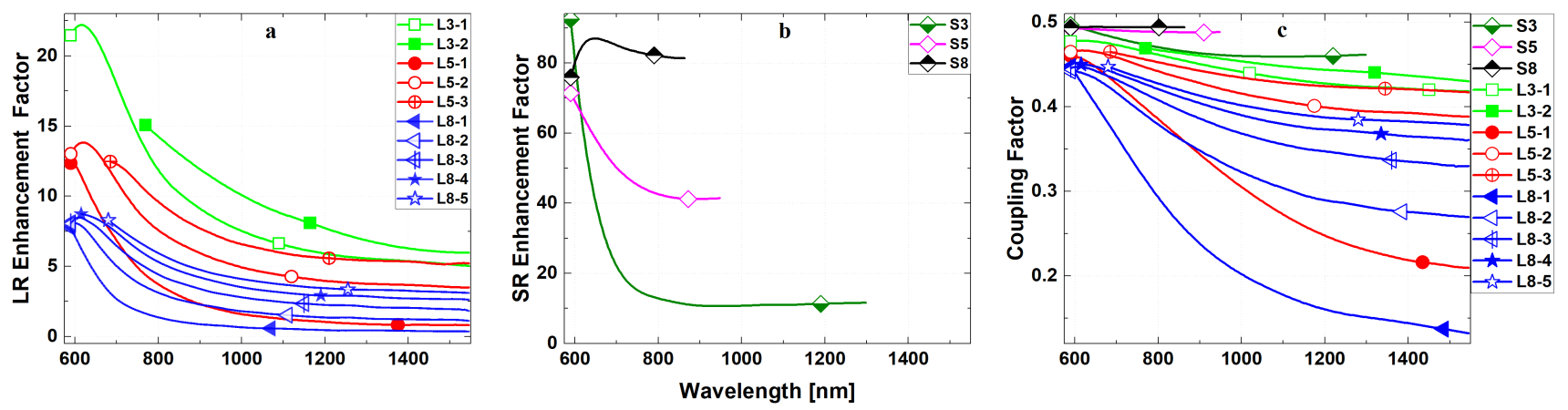

Figure 3: Decay rate enhancement factor of electric dipole emission in vicinity of HMM slabs having 3, 5 and 8 periods, coupled to the a) LR and b) SR high-k modes. Slabs with 3, 5 and 8 periods have 2 (green lines, square), 3 (red lines, circle) and 5 (blue lines, triangle-asterisk) LR modes respectively, and 1 SR (diamonds) mode. c) Coupling factor of the dipole emission into the individual SR and LR modes.

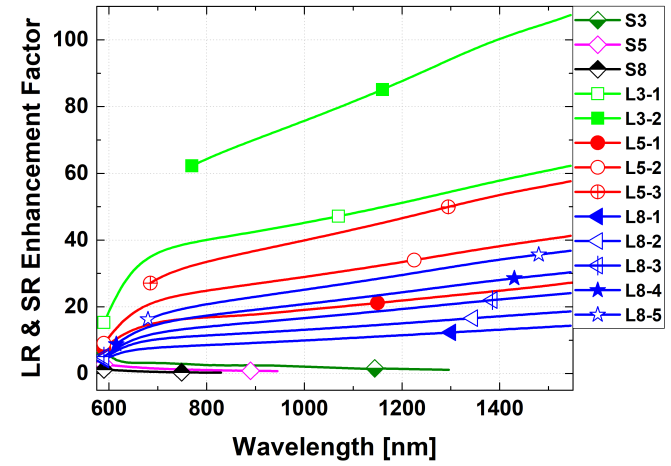

Figure 4: Decay rate enhancement factor of a dipole placed inside the HMM slabs, coupled to individual LR and SR high-k modes. Slabs with 3, 5 and 8 periods have 1 SR and 2, 3 and 5 LR modes respectively.

$$
C F=\frac{\gamma_{w g}(\omega)}{\gamma_{\text {total }}(\omega)}=\frac{\gamma_{w g}(\omega)}{\gamma_{\text {iso }}(\omega)+\gamma_{w g}(\omega)}
$$

The modal LDOS is necessary to be investigated for the two classes of high-k modes in any arbitrary multilayer HMMs. But, from practical point of view, the transition rate enhancement factor is defined by contribution of all available modes in the HMMs. Therefore, we introduce the total enhancement factor, $T E F$, as

$$
T E F=\frac{\sum_{L R}\left[E F_{j n} \times C F_{j n}\right]+\sum_{S R}\left[E F_{n} \times C F_{n}\right]}{\sum_{L R}\left[C F_{j n}\right]+\sum_{S R}\left[C F_{n}\right]}
$$

summing over enhancement factor of each mode weighted by its coupling factor, where $E F_{n j}$ and $C F_{n j}$ are enhancement and coupling factors of $j$ th LR mode of the HMM slab having $n$ period. Correspondingly, $E F_{n}$ and $C F_{n}$ are enhancement and coupling factors of SR modes of the corresponding slab having $n$ period.

\subsection{Mode-resolved Directional Emission}

According to the hyperboloid isofrequency surface of $\mathrm{HMMs}$ type II, Poynting vector $S$ lies within a region $\left(\theta_{c} \leqslant \theta<90\right)$ socalled the resonance cone [37]. The ratio between the power flow in the transverse and vertical directions determine the cone's half angle. Based on the nonlocal homogenization and waveguide definition for the HMM slabs, we define the moderesolved half-angle of resonance cone given by

$$
\tan \left(\theta_{c}\right)=\frac{S_{x}}{S_{z}}=\frac{\operatorname{Re}\left(\frac{\beta}{\epsilon_{e}^{n l}}\right)}{\operatorname{Re}\left(\frac{\mathbb{K}_{S R / L R}}{\epsilon_{o}^{n l}}\right)}
$$

One can infer from Eq. 11) that spontaneous emission from a dipole coupled to each high-k mode should have different resonance cone because of its dependency on the effective mode index $\beta$.

\section{Results and Discussion}

To examine the mode-resolved directional enhancement theory, a quantum emitter is chosen as a perpendicularly oriented electric dipole placed inside and in near vicinity of a finite $\mathrm{HMM}$. A configuration comprising a 15-25 nm thick Au and $\mathrm{SiO}_{2}$ layers respectively, are chosen as unit cell of the HMM. Structures consisting of a substrate (silica glass) - multilayer stacks and a superstrate (air) are considered as the HMM slabs. In our recent study, it is proved that HMMs having 3 periods and onward, show less than $5 \%$ mean-square error between modes dispersion of the multilayer structures and their corresponding effective medium response [38]. Therefore, slabs consisting of basic- 3 , intermediate- 5 and higher- 8 number of periods are chosen for the following characterizations. According to the EMA, the corresponding effective medium of such multilayer stacks operate at wavelengths above $\lambda=530 \mathrm{~nm}$ in the hyperbolic regime type II [39] that is called as transition wavelength to the hyperbolic regime.

High-k modes dispersion calculations shows 1 SR and 2, 3 and 5 LR high-k modes in the HMMs having 3, 5 and 8 periods, respectively as shown in Fig. 2(a-c). The green dashed lines denote the SR mode, blue and red dashed lines represent the odd and even LR modes [24]. LR propagating modes possess high effective indices, on the other side, the SR mode has the lowest effective index fading as a wideband mode for thicker HMMs. Mode calculations of the multilayer HMMs are also carried out by the Transfer Matrix Method (TMM) and shown in the background of Fig. 2, for comparison. In TMM approach, dips in 

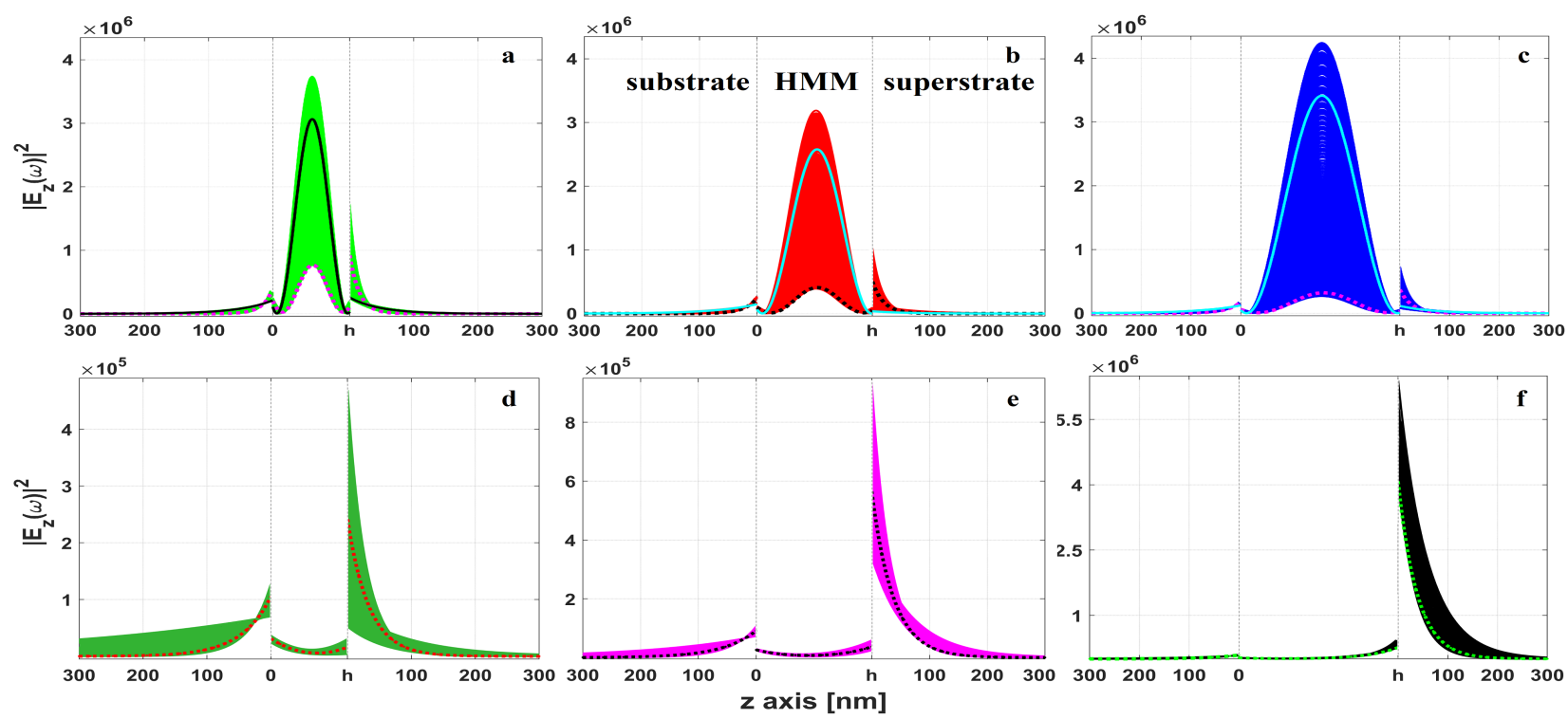

Figure 5: Electric field energy density of the first LR (1st row) and the SR ( 2 nd row) modes in HMM slabs having 3 (a, d), 5 (b, e) and 8 (c, f) periods and in 300 nm distance from the slab into substrate (left side) and superstrate (right side), calculated over all bandwidth. The dotted lines highlight electric field energy density at wavelength $630 \mathrm{~nm}$ and the solid line highlight that of $1400 \mathrm{~nm}$.

the reflection spectrum correspond to the modes of the multilayer structures. Figure 2 depicts a perfect matching between ${ }_{160}$ the theoretical and numerical methods. By adding more periods to the HMM slabs number of LR modes gets increased and their effective index shifts to the lower values. Nonlocal effects and boundary conditions induce an upper cut-off for the wave vector and limits the number of possible LR modes [24]. As number of high-k modes in an HMM slab is finite, LDOS of the HMMs would be finite as well.

QE placed outside HMMs. For a dipole placed outside the HMMs, $10 \mathrm{~nm}$ above in the superstrate, the mode-resolved transition rate enhancement factor is calculated and shown $n_{170}$ in Fig. 3(a). The coupled emission into the individual high-k modes indicates that LR modes of the 3-period slab provide the highest enhancement amongst all three configurations. The EF of the 5-period slab is also greater than that of the 8-period slab. Fig. 3 (b) illustrates that SR modes enhancement factor of the 8-period slab is the strongest amongst the 3 and 5-period HMMs in the total bandwidth. Coupling factor of each LR and SR modes of the 3, 5 and 8-period HMM slabs are shown in fig. 3(c). It is clear that the SR modes have the strongest coupling factor around $50 \%$. While wideband LR modes have strong cou-180 pling near the transition wavelength to the hyperbolic regime, epsilon-near-zero (ENZ) zone; they do not preserve the same coupling factor in the wideband and CF get decreased in tails of the modes.

QE placed inside HMMs. The enhancement factor calculations for a QE placed inside the HMMs are depicted in Fig. 4 It indicates that LR modes generally provide much higher enhancement factors than SR modes of the HMM slabs. On the contrary to the previous case, EF increases far from transition wave-190 length, in tail of the wideband long-range modes. But in the same way, the 3-period HMM slab provides the strongest EF amongst all three configurations. To elaborate on the moderesolved enhancement factors, following discussions should be taken into considerations.

\subsection{Modal Energy Density Distribution.}

It is clear from Eq. (7a) and Eq. (8) that the transition rate of a dipole emission into the HMM slab modes is directly proportional to electric field energy density in the location of the dipole $\left|E_{e d}(z, \omega)\right|^{2}$ and inversely proportional to the volume energy density in the slab and surroundings, $|E(z, \omega)|^{2}$. For slabs having 3, 5 and 8 periods the electric field intensity in the HMM slabs and surroundings are calculated for the first LR and the SR modes and depicted in fig. 5(a-c), and 5 (d-f), respectively. It can be deduced from Fig. 5(a-c) that field intensity of the LR propagating modes is intense and localized inside the slabs. Outside fields intensity, in the substrate and superstrate, is much weaker and clearly decaying. On the contrary to the LR modes, the SR modes have stronger field intensity in the surroundings than in the slabs, inferred from fig. 5 (d-f). The maximum field intensity outside in the superstrate is provided by the 8-period slab. The asymmetric surrounding of the slab is distinguished in the field intensities, emphasizing the role of substrate and superstrate materials in modifying field distribution and consequently the LDOS. As it is expected for the first LR modes, there is one antinode (maximum intensity) in the middle of slabs. It should be mentioned that for the second, third and higher order LR modes, respectively, there are two, three and higher number of peaks in the field intensity distribution in the slabs as is described in our previous study [24]. Therefore, the position of emitter inside the slabs is chosen based on the location of maximum electric field energy density (correspondingly the maximum local density of states) of each mode for calculating the modal enhancement factor. The plots in Fig. 5 are 


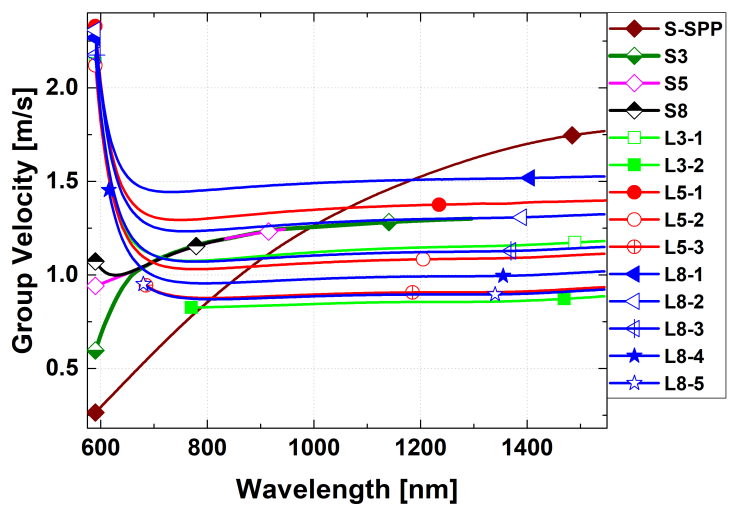

Figure 6: Group velocity of LR and SR modes in HMM slabs consisting of 15-25 $\mathrm{nm}$ thick $\mathrm{Au}-\mathrm{SiO}_{2}$ layers as well as group velocity of the S-SPP mode in $15 \mathrm{~nm}$ thick Au layer. Slabs with 3, 5 and 8 periods have 1 SR and 2, 3 and 5 LR modes, respectively.

calculated over the whole bandwidth of the modes. To take a look at a specific frequency LDOS, two chosen wavelengths are highlighted. The field intensity corresponding to the LDOS at $\lambda=$ $630 \mathrm{~nm}$ is emphasized with the dotted lines in Fig. 5 and that of $\lambda=1400 \mathrm{~nm}$ is highlighted using the solid lines. It is clear that LDOS of the LR modes at the tails $(\lambda=1400 \mathrm{~nm})$ is too strong in the HMM slabs and almost too weak in the surroundings. While at $\lambda=630 \mathrm{~nm}$ (near the transition) LDOS of the LR modes 200 is strong in the surrounding media. The same holds true for SR modes at $\lambda=630 \mathrm{~nm}$. Our findings illustrate the localization of energy by LR high-k modes inside the HMMs, in accordance ${ }^{240}$ to the bulk Bloch waves or correspondingly named Bulk Plasmon Polariton (BPP) modes prediction in HMMs (the LR correspondence) [40]. Furthermore, the results indicate that the SR modes are responsible for localization of energy in nearfield distance outside the HMM slabs, where both classes of modes, in terms of number and effective mode index, are related to the number of periods.

\subsection{Modal Group Velocity.}

Another important parameter affecting the transition rate enhancement is the group velocity of high-k modes. Equation (7) illustrates that the lower the group velocity of the mode is,

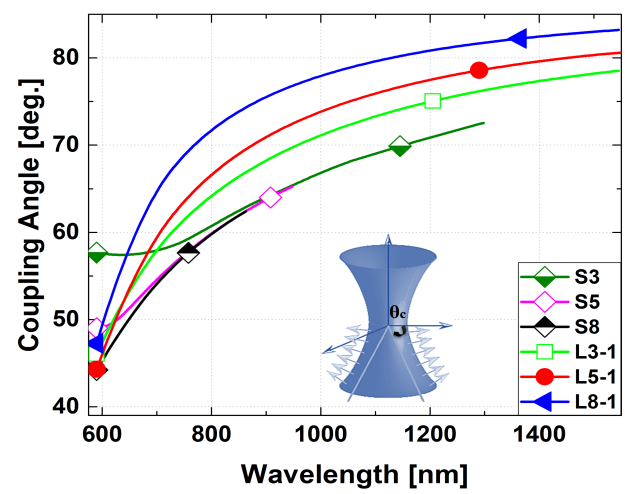

Figure 7: Coupling angle of the SR and first LR high-k modes in HMM slabs consisting of 3,5 and 8 periods of $\mathrm{Au}-\mathrm{SiO}_{2}$ layers working as a $\mathrm{HMM}$ type II. the bigger the LDOS associated with it would be. Since $V_{g r}$ can be thought as the velocity at which energy is conveyed, the modes having slower group velocities provide stronger lightmatter interaction and consequently larger LDOS. Group velocities of SR and LR modes of HMM slabs having 3, 5 and 8 periods, depicted in Fig. 6, indicate that LR modes possess high group the transition wavelength, decreasing on the tails of modes. But the SR mode has a lower group velocity near the transition point increasing at longer wavelengths. Therefore, near the transition wavelength, SR modes will dominate in providing the large LDOS, but far from the ENZ regime, the broadband LR high-k modes will represent the larger LDOS. In the considered HMM slabs, the mode number 2 of the 3-period HMM that exhibits the lowest group velocity has the maximum enhancement factor amongst the others, shown in Fig. 3(a).

Hence, in order to engineer LDOS in HMM slabs the number of periods in the HMMs, working frequencies as well as the emitter location will be of essential matters. For wavelengths near the transition, the SR modes play the key role in transition rate enhancement of QEs placed outside the HMM slabs. Inversely, for QEs located inside the HMM slabs and far from the transition zone, LR high-k modes need to be engineered for manipulating the transition rate enhancement.

\subsection{Modal Emission Direction}

A half-angle of preferential emission direction of a dipole into the SR and first LR modes of the HMM slabs with 3, 5 and 8 periods is shown in Fig. 7. For HMMs type II, the bigger $\theta_{c}$ results in energy flow closer to the surface of slabs as it is obvious in the iso-frequency surface depicted in the inset of Fig. 7. Energy coupling to the SR and LR high-k modes of the HMM slabs occurs in a small and almost the same emission cones at wavelengths near the transition zone. Moving farther form the ENZ, emission coupled to different modes starts to diverge. In longer wavelengths, energy mostly flows near the surface of the slab because of the high coupling angles for both the LR and SR modes, yet the LR modes show higher coupled angles in comparison to the SR mode. A small emission cone at wavelengths near the transition zone, for both SR and LR modes indicate the best working frequencies. In near-fields applications, emission at angles close to the vertical direction will be desirable. Using objectives, the more the vertical emission is, the lower the objective aperture can be used. For bigger half angles emission direction falls out of cones aperture and reduces the photon collection. Therefore, working at shorter wavelengths (near the transition point) will be more desirable. It is worth mentioning that emission outcoupling to far-field needs some approaches to provide k-matching conditions 41. Modes having lower effective indices will make the outcoupling more facile. Accordingly, the SR modes having lower effective index, have advantages over LR high-k modes close to the transition point and even far from it.

\subsection{Thin vs. thick HMMs.}

Investigation on individual high-k modes of the HMM slabs indicates that the modes related to thinner slabs provide larger 

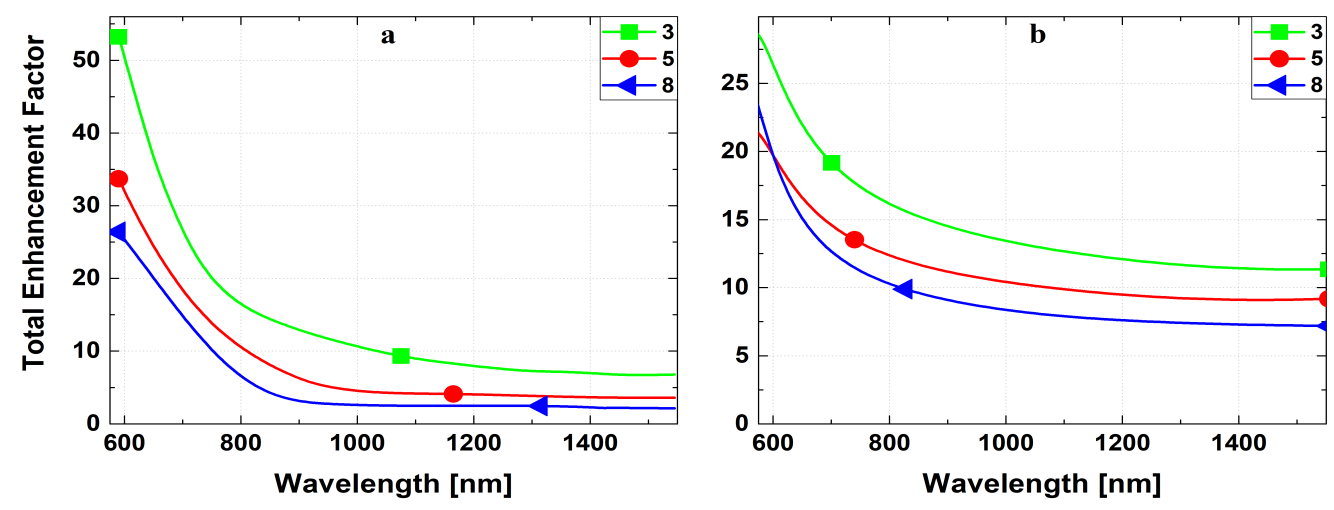

Figure 8: a) Theoretical and b) simulation calculation of total decay rate enhancement factor of electric dipole in vicinity of HMM slabs having 3, 5 and 8 periods; summing over contribution of the SR and all LR high-k modes.

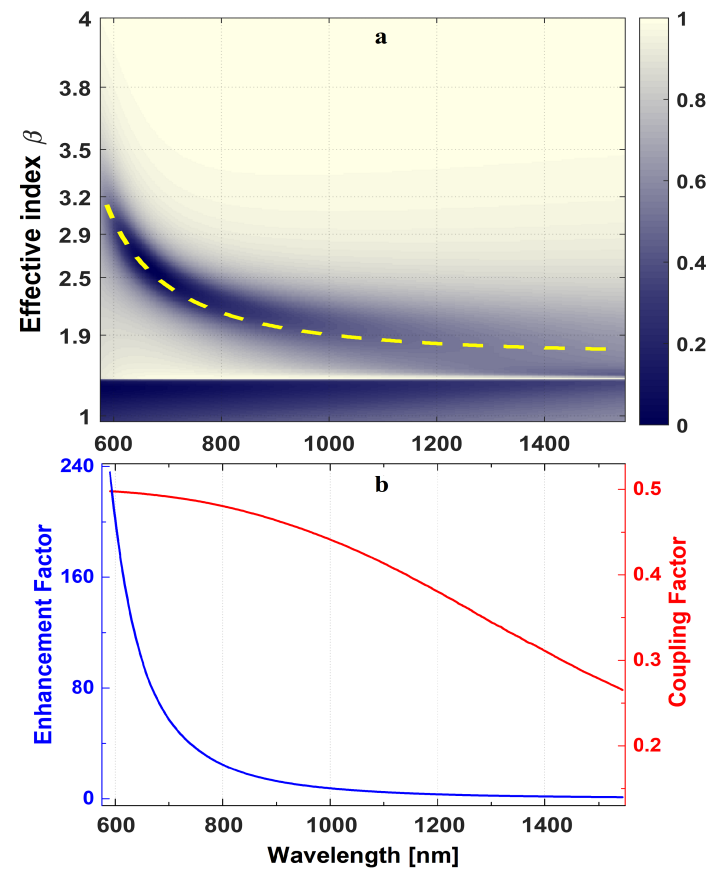

Figure 9: a) Short range surface plasmon polariton (S-SPP) dispersion of a single $\mathrm{Au}$ layer of $15 \mathrm{~nm}$ thick $\mathrm{Au}$ sandwiched between $\mathrm{SiO}_{2}$ substrate and air superstrate. b) Transition rate enhancement and coupling factor for an electric dipole placed $10 \mathrm{~nm}$ above the metal layer in superstrate.

LDOS. For HMM slabs with 3, 5 and 8 periods the total enhancement factor for a dipole in the vicinity of HMM slabs, based on the eq. 10 , is also calculated and shown in Figure $8(a) . I_{31}$ proves that multilayer stacks with a lower number of periods provide the higher total enhancement factor. Larger number of modes in a thick slab (having multiple nodes and antinodes in the slab) facilitates their competition, which leads to the certain degradation of local density of states. To validate the theoretical calculations, we conducted a 3D FDTD simulation using commercially available Lumerical software. Simulation results shown in figure 8(b) affirm our theoretical derivations with the same conclusion. It is worth mentioning that the TEF calculations of a dipole outside the slabs indicate the maximum ${ }_{320}$ enhancement appears near the transition wavelength, in total accordance with the above mode-resolved enhancement factor calculations as well as the experimental results reported in literature [29, 42, 43].

Investigation on LDOS of HMM slabs indicating the strongest transition rate enhancement is attached to the structures with smaller number of periods, raises a question about preferences of using a single metal layer (SML) instead of multilayer stacks. To provide a comparison, we considered a single Au layer with $15 \mathrm{~nm}$ thickness on a glass substrate, placed in free space (air as superstrate), the same as HMM slabs configurations. Looking for modes of the metal layer using TMM and theoretical calculation, we realize only one mode having high wave vector, shown in Fig. 9(a), familiar as a short range surface plasmon polariton (S-SPP). The enhancement factor as well as coupling factor of a dipole placed $10 \mathrm{~nm}$ above the layer, are calculated and shown in figure 9 (b). In comparison to enhancement factor of SR mode in the 3-period HMM shown in figure 3(b), the single metal layer mode shows higher enhancement factor. Although coupling factor of the S-SPP at shorter wavelengths is comparable to that of the SR mode, it decreases by increasing the wavelength and seems weaker compared to the high-k SR mode. Group velocity of the S-SPP mode in SML is shown in Fig. 6 to be easily comparable to that of the high-k modes in the HMM slabs. It shows a lower group velocity at shorter wavelengths increasing fast at the longer wavelengths (decreasing LDOS), where the enhancement factor of the mode drops quickly.

It is clear that the high-k SR mode possesses lower effective index than the SML mode. As dispersion of the SR-SPPs in a single metal layer has lower effective index in comparison to a pure surface plasmon polariton (a bulk metal), adding more layers, the coupled SPPs shifts more to lower effective indices. Since the in/out coupling of energy to a structure's mode in addition to LDOS engineering is important and very promising for improvement of numerous near-field technologies, HMM structures hold advantages over SML in this regard. Besides that, the transition wavelength to the hyperbolic regime with the maximum enhancement factor for $\mathrm{HMMs}$, can be tuned easily by engineering the unit cell; while such tunability for SMLs is not available [44]. It also should be noted that the max- 
imum LDOS not necessarily gives rise to the maximum radiative emission enhancement for QEs. In transition rate enhance-380 ment, the portion of radiative and nonradiated LDOS needs to be considered separately, based on the application. For the SR-SPP mode existing at the metal-dielectric interfaces, there is stronger damping and correspondingly nonradiative transitions for a QE placed close to the metal rather than HMMs that behaves like dielectrics in two direction[21].

\section{4. conclusion}

In this study, a mode-resolved photonic local density of states and energy flow directionality of finite thickness HMM slabs are investigated. The results demonstrate that the LDOS enhancement elucidated through a quantum emitter coupled to the high-k modes of HMM slabs, is indeed totally dependent on the number of periods in HMMs as well as position ${ }^{400}$ of the emitter. The introduced SR and LR high-k modes play the most important role in LDOS engineering inside and in the near vicinity of the HMM slabs, respectively. In both positions,

340 HMM slabs having smaller number of periods show stronger transition rate enhancement near the transition wavelength to the hyperbolic regime. Furthermore, SR high-k modes show lower coupling angles for directional emission of the coupled quantum emitters. In general, a low-period multilayer meta- ${ }^{410}$ material seems promising for the hyperbolic LDOS engineering and optimal directional emission of quantum emitters. The results confirm that applicability of hyperbolic metamaterials in spontaneous emission enhancement is dependent on fully understanding of high-k modes of the HMMs to properly engineer application-oriented HMM slabs.

\section{References}

[1] L. Lu, R. E. Simpson, S. K. Valiyaveedu, Active hyperbolic metamaterials: Progress, materials and design, J. Opt. 20 (10) (2018) 103001. doi:10. 1088/2040-8986/aade68

[2] M. Kim, S. So, K. Yao, Y. Liu, J. Rho, Deep sub-wavelength nanofocusing of UV-visible light by hyperbolic metamaterials, Sci. Rep. 6 (2016) 38645. doi:10.1038/srep38645

[3] K. V. Sreekanth, Y. Alapan, M. ElKabbash, E. Ilker, M. Hinczewski, U. A. Gurkan, A. De Luca, G. Strangi, Extreme sensitivity biosensing platform based on hyperbolic metamaterials. Nat. Mater. 15 (6). doi:10.1038/ nmat4609 URL http://www . nature.com/doif inder/10.1038/nmat4609

[4] M. A. Baqir, A. Farmani, T. Fatima, M. R. Raza, S. F. Shaukat, A. Mir, Nanoscale, tunable, and highly sensitive biosensor utilizing hyperbolic metamaterials in the near-infrared range, Appl. Opt. 57 (31) (2018) 94479454.

[5] X. Yin, H. Zhu, H. Guo, M. Deng, T. Xu, Z. Gong, X. Li, Z. H. Hang, C. Wu, H. Li, S. Chen, L. Zhou, L. Chen, Hyperbolic Metamaterial Devices for Wavefront ${ }_{440}$ Manipulation, Laser Photonics Rev. 13 (2018) 1800081.

370 [6] M. S. Habib, A. Stefani, S. Atakaramians, S. C. Fleming, B. T. Kuhlmey, Analysis of a hyperprism for exciting high- $\mathrm{k}$ modes and subdiffraction imaging. Phys. Rev. B 100 (2019) 115146. URL https://doi.org/10.1103/PhysRevB.100.115146

[7] T. Repan, S. Zhukovsky, A. Lavrinenko, M. Willatzen, Dark-field hyperlens for high-contrast sub-wavelength imaging. Vol. 9883, 2016, p. 98830T. doi:10.1117/12.2224720

1] URL http://proceedings.spiedigitallibrary.org/ proceeding. aspx?doi=10.1117/12.2224720
[8] D. Lu, H. Qian, K. Wang, H. Shen, F. Wei, Y. Jiang, E. E. Fullerton, P. K. $\mathrm{Yu}, \mathrm{Z}$. Liu, Nanostructuring Multilayer Hyperbolic Metamaterials for Ultrafast and Bright Green InGaN Quantum Wells, Adv. Mater. 30 (15) (2018) 1706411. doi:10.1002/adma.201706411

n[9] T. Galfsky, J. Gu, E. E. Narimanov, V. M. Menon, Photonic hypercrystals for control of light-matter interactions. Proc. Natl. Acad. Sci. U. S. A. 114 (20) (2017) 5125-5129. doi : 10.1073/pnas .1702683114 URL http://www.pnas.org/lookup/doi/10.1073/pnas. 1702683114

[10] Z. Jacob, I. I. Smolyaninov, E. E. Narimanov, Broadband Purcell effect: Radiative decay engineering with metamaterials, Appl. Phys. Lett. 100 (18) (2012) 181105. doi:10.1063/1.4710548

[11] S. V. Zhukovsky, O. Kidwai, J. E. Sipe, Physical nature of volume plasmon polaritons in hyperbolic metamaterials, Opt. Express 21 (12) (2013) 14982. doi:10.1364/OE.21.014982

[12] L. Novotny, B. Hecht, Principles of Nano-Optics, Cambridge university Press, 2006.

[13] V. Drachev, V. Podolskiy, A. Kildishev, Hyperbolic metamaterials: new physics behind a classical problem, Opt. Express 21 (12) (2013) 16991701. doi:10.1364/0E.21.015048

[14] T. Galfsky, H. N. S. Krishnamoorthy, W. Newman, E. E. Narimanov, Z. Jacob, V. M. Menon, Active hyperbolic metamaterials: enhanced spontaneous emission and light extraction, Optica 2 (1) (2015) 62. doi:10.1364/OPTICA.2.000062

II URL https://www.osapublishing.org/abstract.cfm?URI= optica-2-1-62

[15] Z. Jacob, J. Y. Kim, G. V. Naik, A. Boltasseva, E. E. Narimanov, V. M. Shalaev, Engineering photonic density of states using metamaterials, Appl. Phys. B: Lasers Opt. 100 (1) (2010) 215-218. doi:10.1007/ s00340-010-4096-5

[16] V. Caligiuri, R. Dhama, K. V. Sreekanth, G. Strangi, A. De Luca, Dielectric singularity in hyperbolic metamaterials: the inversion point of coexisting anisotropies Sci. Rep. 6 (2016) 20002. doi : 10.1038/srep20002 URL http://www . nature.com/articles/srep20002

[17] L. Ferrari, D. Lu, D. Lepage, Z. Liu, Enhanced spontaneous emission inside hyperbolic metamaterials, Opt. Express 22 (4) (2014) 4301-4306. doi : 10.1364/OE.22.004301

[18] P. Ginzburg, D. J. Roth, M. E. Nasir, P. Segovia, A. V. Krasavin, J. Levitt, L. M. Hirvonen, B. Wells, K. Suhling, D. Richards, V. A. Podolskiy, A. V. Zayats, Spontaneous emission in non-local materials, Light: Sci. Appl. 6 (2017) e16273. doi:10.1038/lsa.2016.273

420 [19] M. A. Noginov, H. Li, Y. A. Barnakov, D. Dryden, G. Nataraj, G. Zhu, C. E. Bonner, M. Mayy, Z. Jacob, E. E. Narimanov, Controlling spontaneous emission with metamaterials., Opt. Lett. 35 (11) (2010) 1863-1865. doi : 10.1364/0L.35.001863

[20] W. D. Newman, C. L. Cortes, Z. Jacob, Enhanced and directional singlephoton emission in hyperbolic metamaterials, J. Opt. Soc. Am. B 30 (4) (2013) 766-775. doi:10.1364/JOSAB.30.000766

[21] P. Shekhar, J. Atkinson, Z. Jacob, Hyperbolic metamaterials: fundamentals and applications, Nano Convergence 1 (2014) 14. doi:10.1186/ s40580-014-0014-6

[22] A. Goncharenko, Y.-C. Chang, R. J. Knize, A. O. Pinchuk, Extraordinary highand low-momentum lossless plasmonic modes in one-dimensional metamaterials, Opt. Mater. Express 7 (3). doi:10.1364/0ME.7.000766

[23] A. A. Orlov, P. M. Voroshilov, P. A. Belov, Y. S. Kivshar, Engineered optical nonlocality in nanostructured metamaterials, Phys. Rev. B 84 (2011) 045424. doi:10.1103/PhysRevB.84.045424

[24] M. Mahmoodi, S. H. Tavassoli, O. Takayama, J. Sukham, R. Malureanu, A. V. Lavrinenko, Existence Conditions of High-k Modes in Finite Hyperbolic Metamaterials, Laser Photonics Rev. 13 (3) (2019) 1800253. doi : 10.1002/lpor.201800253

[25] H. N. S. Krishnamoorthy, Z. Jacob, E. Narimanov, I. Kretzschmar, V. M. Menon, Topological Transitions in Metamaterials., Science 336 (6078) (2012) 205-209. doi:10.1126/science.1219171

[26] A. S. Potemkin, A. N. Poddubny, P. A. Belov, Y. S. Kivshar, Green function for hyperbolic media, Phys. Rev. A 86 (023848). doi:10.1103/PhysRevA. 86.023848

[27] I. Iorsh, A. Poddubny, A. Orlov, P. Belov, Y. S. Kivshar, Spontaneous emission enhancement in metal-dielectric metamaterials, Phys. Lett. A 376 (3) (2012) 185-187. doi:10.1016/j.physleta.2011.11.001

[28] O. Kidwai, S. V. Zhukovsky, J. E. Sipe, Dipole radiation near hyperbolic 

Letters 36 (13) (2011) 2530. doi:10.1364/ol .36.002530

[29] C. L. Cortes, W. Newman, S. Molesky, Z. Jacob, Quantum nanophotonics using hyperbolic metamaterials, J. Opt. 14 (6) (2012) 063001. doi:10. 1088/2040-8978/14/6/063001

[30] A. A. Orlov, P. M. Voroshilov, P. A. Belov, Y. S. Kivshar, Engineered optical nonlocality in nanostructured metamaterials, Phys. Rev. B 84, (2011) 045424. doi:10.1103/PhysRevB.84.045424

[31] V. Popov, A. V. Lavrinenko, A. Novitsky, Operator approach to effective medium theory to overcome a breakdown of Maxwell Garnett approxi460 mation, Phys. Rev. B 94, (2016) 085428. doi:10.1103/PhysRevB.94. 085428

[32] V. Popov, A. V. Lavrinenko, A. Novitsky, Surface waves on multilayer hyperbolic metamaterials: Operator approach to effective medium approximation, Phys. Rev. B 97 (125428).

[33] M. Y. Shalaginov, V. V. Vorobyov, J. Liu, M. Ferrera, A. V. Akimov, A. Lagutchev, A. N. Smolyaninov, V. V. Klimov, J. Irudayaraj, A. V. Kildishev, A. Boltasseva, V. M. Shalaev, Enhancement of single-photon emission from nitrogen-vacancy centers with $\mathrm{TiN} /(\mathrm{Al}, \mathrm{Sc}) \mathrm{N}$ hyperbolic metamaterial, Laser Photonics Rev. 9 (2015) 120-127. doi : 10.1002/lpor. 201400185

[34] K. V. Sreekanth, M. Elkabbash, Y. Alapan, A. R. Rashed, U. A. Gurkan, G. Strangi, A multiband perfect absorber based on hyperbolic metamaterials Sci. Rep. 6 (26272). doi : 10.1038/srep26272 URL http://dx.doi.org/10.1038/srep26272

[35] Y. C. Jun, R. M. Briggs, H. A. Atwater, M. L. Brongersma, Broadband enhancement of light emission in silicon slot waveguides, Opt. Express 17 (9) (2009) 7479. doi:10.1364/oe.17.007479

[36] Y. C. Jun, R. D. Kekatpure, J. S. White, M. L. Brongersma, Nonresonant enhancement of spontaneous emission in metal-dielectric-metal plasmon waveguide structures, Phys. Rev. B 78 (15) (2008) 153111. doi: 10.1103/PhysRevB.78.153111

[37] C. Argyropoulos, N. Estakhri, F. Monticone, A. Alù, Negative refraction, gain and nonlinear effects in hyperbolic metamaterials, Opt. Express 21 (12).

[38] J. Sukham, O. Takayama, M. Mahmoodi, S. Sychev, A. Bogdanov, S. H. Tavassoli, A. V. Lavrinenko, R. Malureanu, Investigation of effective media applicability for ultrathin multilayer structures, Nanoscale 11 (2019) 12582. doi:10.1039/C9NR02471A

[39] L. Ferrari, C. Wu, D. Lepage, X. Zhang, Z. Liu, Hyperbolic metamaterials 490 and their applications, Prog. Quantum Electron. 40 (2015) 1-40. doi: 10.1016/j.pquantelec.2014.10.001

[40] I. Avrutsky, I. Salakhutdinov, J. Elser, V. Podolskiy, Highly confined optical modes in nanoscale metal-dielectric multilayers, Phys. Rev. A 75 (2007) 241402. doi:10.1103/PhysRevB.75.241402

[41] K. V. Sreekanth, A. De Luca, G. Strangi, Excitation of volume plasmon polaritons in metal-dielectric metamaterials using $1 \mathrm{D}$ and 2D diffraction gratings, J. Opt. 16 (10) (2014) 105103. doi:10.1088/2040-8978/16/ $10 / 105103$

[42] A. N. Poddubny, P. A. Belov, Y. S. Kivshar, Spontaneous radiation of a finitesize dipole emitter in hyperbolic media, Phys. Rev. A 84 (2011) 023807. doi:10.1103/PhysRevA.84.023807

[43] A. V. Chebykin, A. A. Orlov, A. S. Shalin, A. N. Poddubny, P. A. Belov, Strong Purcell effect in anisotropic $\epsilon$-near-zero metamaterials, Phys. Rev. B 91 (2015) 205126. doi:10.1103/PhysRevB.91.205126

[44] P. Ben-abdallah, K. Joulain, J. Drevillon, G. Domingues, Tailoring the local density of states of nonradiative field at the surface of nanolayered materials, Appl. Phys. Lett. 94 (2009) 153117. doi:10.1063/1.3122139 